\title{
Mieloma múltiple en Chile. Características clínicas y sobrevida
}

\author{
Guillermo Conte $L^{1,7}$, Gastón Figueroa $M^{1}$, Vivianne Lois $V^{2}$, \\ María Elena Cabrera $C^{3}$, Alvaro León $\mathbf{R}^{4}$, Hernán $\mathbf{G}$ arcía $\mathbf{L}^{5}$, \\ Hernán Rojas $\mathbf{R}^{6}$.

\section{Clinical features and survival of Chilean patients with multiple myeloma}

Background: Mortality rate records are the only data available in Chile about the prognosis of patients with multiple myeloma (MM). Aim: To characterize clinical features, survival rate and factors related to mortality in cases with MM treated in six large medical centers in Chile. Material and Method: Retrospective analysis of demographic data, clinical features and survival rate records of patients with MM, collected between 1998 and 2002. Survival curves were generated and a multivariate analysis of factors associated to early mortality was carried out. Results: Data from 245 patients aged 38 to 95 years (129 women) was collected. Fifty two percent had an IgG myeloma, 25\% had and IgA and 6.1\% had light chains myeloma. According to Durie and Salmon staging system, $8,2 \%$ were in Stage I $12.6 \%$ in Stage II, $60.5 \%$ in Stage III and in $18.8 \%$ the information about staging was not available. Fifty percent had an hemoglobin level below $10 \mathrm{~g} / \mathrm{dL}, 30 \%$ had a serum creatinine over $2 \mathrm{mg} / \mathrm{dL}$ and $28 \%$ had a serum calcium level over $10.5 \mathrm{mg} / \mathrm{dL}$ Median survival was 33 months. Twenty percent of patients died within the first six months after diagnosis (early mortality). Predictive factors for early mortality were male sex, thrombocytopenia, anemia, renal failure, hypercalcemia, a beta2-microglobulin $\geq 5.5 \mathrm{mg} / \mathrm{L}$ and a serum albumin level $<3.5 \mathrm{~g} / \mathrm{dL}$ There was a correlation between the number of bad prognosis factors present and the probability of early mortality. Conclusions: This group of Chilean patients with MM presented a short survival time, and $20 \%$ died within the first six months after diagnosis. More than a half of cases were diagnosed at an advanced stage (Durie and Salmon Stage III). Several factors were associated to early mortality, two of which (beta 2microglobulin and serum albumin), are included in the new International Staging System for MM (Rev Méd Chile 2007; 135: 1111-17).

(Key w ords: Multiple myeloma; Myeloma proteins; Neoplasm staging; Survival rate)

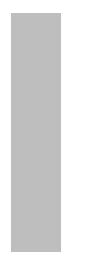

\footnotetext{
Recibido el 4 de septiembre, 2006. Aceptado 4 abril, 2007.

Financiamiento parcial de International Myeloma Foundation. Esta organización no tuvo participación en el diseño del estudio, en la recolección, análisis o interpretación de los datos, ni en la preparación y revisión de manuscrito.

${ }^{1}$ Hospital Clínico de la Universidad de Chile. ${ }^{2}$ Hospital Barros Luco T. ${ }^{3}$ Hospital del Salvador. ${ }^{4}$ Universidad Austral, Hospital Regional de Valdivia. ${ }^{5}$ Hospital San Borja Arriarán. ${ }^{6}$ Hospital Sótero del Río. ${ }^{7}$ International Myeloma Foundation Latin America.
}

Correspondencia a: Dr. Guillermo Conte L. Sección de Hematología, Hospital Clínico Universidad de Chile. Santos Dumont 999, Independencia, Santiago, Chile. Fax: 7777618.

E mail: gfconte@gmail.com 
$\mathrm{E}^{1}$ mieloma múltiple (MM) es una enfermedad caracterizada por la proliferación clonal de células plasmáticas y la presencia de una paraproteína en el suero, orina o en ambas. Sus manifestaciones clínicas son hipercalcemia, insuficiencia renal, anemia y lesiones osteolíticas ${ }^{1}$. La incidencia reportada internacionalmente varía de 0,2 a 5,1 casos x 100.000 habitantes-año ${ }^{2}$, correspondiendo a $1 \%-2 \%$ de todas las neoplasias y a $10 \%$ de las neoplasias hematológicas. En la actualidad no existen estudios sobre la epidemiología del $\mathrm{MM}$ en Chile y los únicos datos disponibles corresponden a las tasas de mortalidad correspondientes al periodo 1985-1999 (Fuente: Minsal http://epi.minsal.cl/).

El pronóstico de los pacientes con MM es muy variable, con una mediana de sobrevida de 2,5 a 3 $a_{\text {ños }}^{3}$. A pesar de la aparición de importantes avances terapéuticos ${ }^{4}$, la enfermedad continúa siendo incurable y hay una importante proporción de pacientes que fallecen precozmente luego del diagnóstico ${ }^{5}$.

Formando parte de un estudio epidemiológico latinoamericano auspiciado por la International Myeloma Foundation, del cual ya existen reportes iniciales desde Brasil ${ }^{6}$, se inició este estudio con el apoyo de la Sociedad Chilena de Hematología, el grupo PANDA (Programa Adulto Nacional de Drogas Antineoplásicas), la Universidad de Chile y la Universidad Austral de Chile (Valdivia), con los objetivos de conocer la epidemiología del MM en Chile y describir la sobrevida de los pacientes con esta patología.

\section{MATERIAL Y MÉTODO}

Este es un estudio retrospectivo, multicéntrico, en el cual participaron 6 centros hospitalarios. Cinco hospitales correspondían a distintas áreas de Santiago: Hospital del Salvador (área oriente), Hospital Barros Luco Trudeau (área sur), Hospital San Borja Arriarán (área centro), Hospital Clínico de la Universidad de Chile (área norte), Hospital Sótero del Río (área sur). El otro centro participante fue el Hospital de Valdivia, vinculado a la Universidad Austral de Chile.

En todos los casos, el diagnóstico de MM se fundamentó en la presencia de una paraproteína en plasma u orina y un porcentaje de células plasmáticas superior a $10 \%$ en la médula ósea ${ }^{7}$.
Se utilizó una hoja de registro diseñada previamente, en la que cada centro recolectó, en forma retrospectiva, información clínica, de laboratorio y sobrevida de los pacientes diagnosticados y tratados entre los años 1988 y 2002. A cada centro se le solicitó registrar la etapa de la enfermedad según el método de Durie y Salmon $(D \& S)^{8}$.

La sobrevida de los pacientes se calculó como mediana de sobrevida en meses y se generaron curvas de sobrevida con el método de Kaplan Meier.

Para el análisis de factores asociados a mortalidad, se dividió a los pacientes en dos grupos según el tiempo de sobrevida (menor o mayor a 6 meses, explicado en Resultados) y se compararon las variables categoriales y continuas con el test de Chi cuadrado y la prueba no paramétrica de Mann-Whitney, respectivamente.

Para la realización de las pruebas estadísticas y generación de las curvas de sobrevida, se utilizó el programa estadístico SPSS 13.0 (SPSS Inc. Chicago, Illinois, USA).

\section{RESUlTADOs}

Se recolectó información de 245 pacientes. La mediana de edad de los pacientes fue 66 años, 18 pacientes $(7,3 \%)$ eran menores de 50 años de edad y 21 pacientes $(8,6 \%)$ mayores de 80 años de edad. La relación de hombres y mujeres fue 1:1,1. Las características demográficas y clínicas de los pacientes al momento del diagnóstico de MM se presentan en la Tabla 1, destacando una elevada frecuencia de anemia (50\%), hipercalcemia (28\%) e insuficiencia renal (30\%). La caracterización del tipo de inmunoglobulina monoclonal reveló que la mayoría de los casos corresponde a IgG $(51,8 \%)$, seguido de IgA $(25,3 \%)$ y cadenas livianas $(6,1 \%)$, fueron raros los tipos $\operatorname{IgM}$ e IgD.

Del total de pacientes, 8,2\% de los pacientes se encontraba en etapa I de D\&S, $12,6 \%$ en etapa II y $60,5 \%$ en etapa III al momento del diagnóstico. En 46 pacientes $(18,8 \%)$ no se contó con información respecto a la etapa según $\mathrm{D} \& \mathrm{~S}$.

En 214 casos se obtuvo información de sobrevida (87\%). La mediana de sobrevida del total de pacientes fue de 33 meses, con una sobrevida a 5 años de $23 \%$. El análisis de la curva de sobrevida (Figura 1), evidencia una mayor mortalidad duran- 
Tabla 1. Características demográficas y clínicas de los casos de mieloma múltiple

\begin{tabular}{|lcc|}
\hline & $\mathrm{n}$ & $\%$ \\
\hline Total de pacientes & 245 & 100 \\
Hombre/mujer & $116 / 129$ & $47 / 53$ \\
Edad mediana (rango) & $66(38-95)$ & \\
Anemia (Hb $<10 \mathrm{~g} / \mathrm{dL})$ & $115 / 226$ & 50,9 \\
Hipercalcemia $(>10,5 \mathrm{mg} / \mathrm{dL})$ & $54 / 189$ & 28,6 \\
Insuficiencia renal (creat $>2 \mathrm{mg} / \mathrm{dL})$ & $67 / 220$ & 30,5 \\
En programa de diálisis & $19 / 220$ & 8,6 \\
Trombocitopenia $(<100.000 / \mu \mathrm{L})$ & $17 / 208$ & 8,2 \\
Tipo de inmunoglobulina & & 51,8 \\
IgG & 127 & 25,3 \\
IgA & 62 & 6,1 \\
Cadenas livianas & 15 & 1,2 \\
IgM & 3 & 0,4 \\
IgD & 1 & 15,1 \\
Sin información & 37 & 8,2 \\
Etapa según D\&S $(\%)$ & & 2 \\
Ia & 20 & 37,6 \\
IIa & 26 & 22,9 \\
IIb & 5 & 18,8 \\
IIIa & 92 & \\
IIIb & 56 & \\
Sin información & 46 & \\
\hline
\end{tabular}

Creat: creatininemia

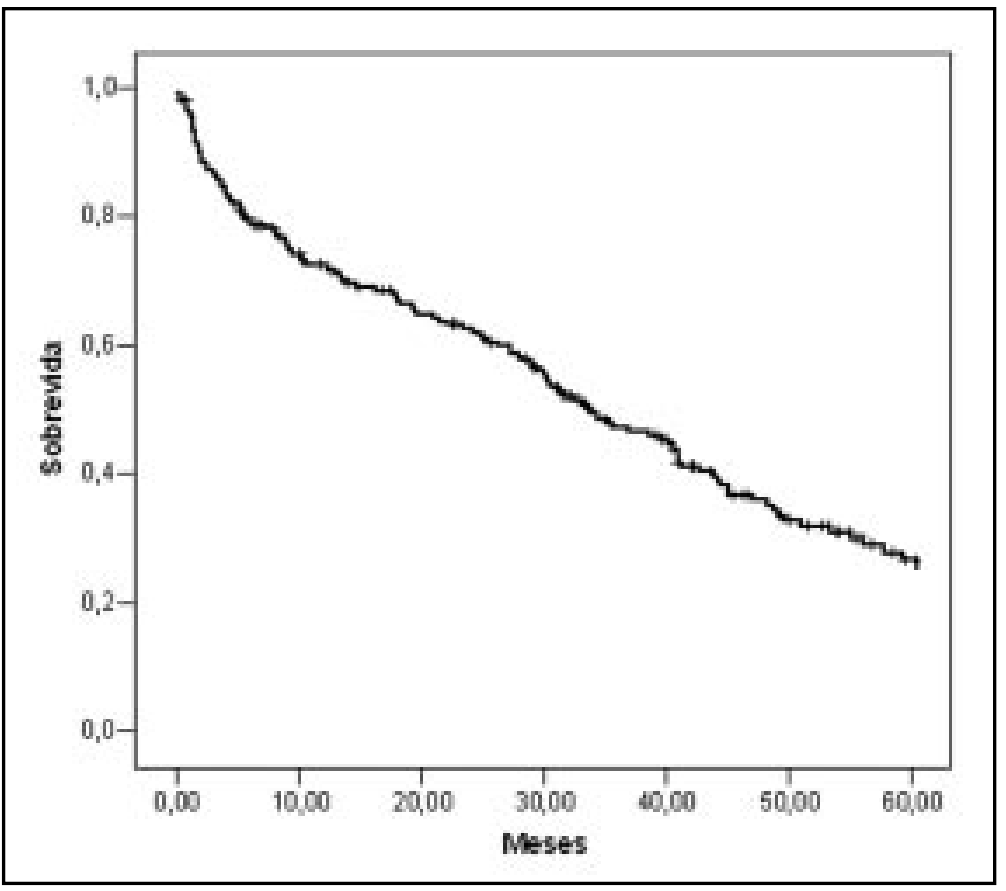

Figura 1. Curva de sobrevida del total de pacientes con mieloma múltiple (n: 214). 
te los primeros meses luego del diagnóstico. El mayor número de muertes ocurrió dentro de los primeros 6 meses desde el diagnóstico $(n=43$; $20 \%$ ), la mitad de las cuales ocurrieron en los 2 primeros meses ( $\mathrm{n}=22 ; 10,2 \%$ ). Después del sexto mes, la tasa de mortalidad se mantuvo estable.

La sobrevida de los pacientes según la etapa de D\&S se presenta en la Figura 2.

Con el fin de identificar factores relacionados a menor sobrevida, realizamos un análisis univariable con los datos obtenidos y evaluamos su asociación con mortalidad precoz, que definimos como la que ocurre dentro de los primeros 6 meses desde el diagnóstico.

Este análisis (Tabla 2) reveló que predicen una mortalidad precoz una serie de características (sexo masculino, anemia, hipercalcemia, insuficiencia renal, trombocitopenia, valores elevados de beta2-microglobulina e hipoalbuminemia). El número de estos factores presentes en cada paciente se correlacionó con una mayor probabilidad de fallecer antes de 6 meses, llegando a una mortalidad precoz de $55 \%$ cuando se encontraban 40 más factores (Figura 3).

\section{Discusión}

En Chile, los únicos datos epidemiológicos directos sobre MM corresponden a las tasas de mortalidad entre los años 1985 y 1999. Durante este periodo se observa un permanente incremento en las tasas de mortalidad (Fuente: Minsal http:// epi.minsal.cl/), que en el año 1999 llegó a 2,0 x 100.000 habitantes-año en los hombres y 2,3 x 100.000 habitantes-año en las mujeres. La International Agency for Research on Cancer (OMS) estima una incidencia de MM en Chile (basado en datos de mortalidad) de 2,2 x 100.000 habitantesaño, lo que representa unos 330 casos nuevos cada año ${ }^{2}$. Se ha observado un aumento en la incidencia de MM a nivel mundial, que puede estar en relación al aumento en la media de edad observada en diversas poblaciones ${ }^{9}$.

Destaca en esta serie de pacientes la alta proporción de pacientes con edades inferiores a 50 años (7,3\%), lo cual discrepa de lo informado clásicamente en la literatura ${ }^{3}$, pero que es coincidente con una reciente publicación de la Clínica Mayo $^{10}$, que reportaron $10 \%$ de pacientes meno-

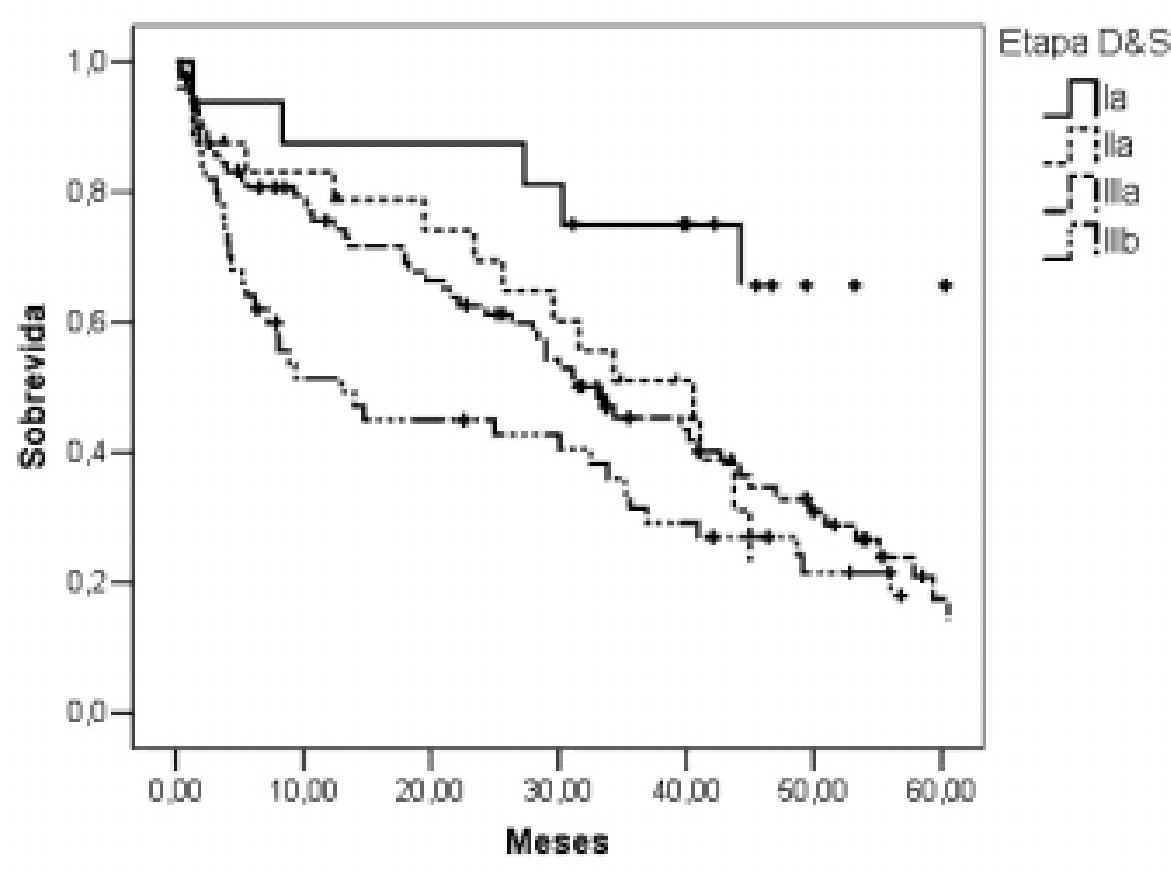

\section{Sobrevida (mediana) 67 meses 40 meses 33 meses 13 meses}

Figura 2. Curvas de sobrevida de los pacientes con mieloma múltiple según etapa de Durie y Salmon. 
Tabla 2. Comparación de las características clínicas de los pacientes con M M que fallecieron precozmente con los que sobrevivieron más de 6 meses

\begin{tabular}{|c|c|c|c|}
\hline \multirow[b]{2}{*}{ Característica } & \multicolumn{2}{|c|}{ Sobrevida } & \multirow[b]{2}{*}{$\mathrm{p}$} \\
\hline & $\begin{array}{c}<6 \text { meses } \\
n: 43\end{array}$ & $\begin{array}{c}>6 \text { meses } \\
\mathrm{n}: 171\end{array}$ & \\
\hline Edad años (rango) & $65(42-93)$ & $66(38-89)$ & NS \\
\hline Edad >65 años & $20(46,5 \%)$ & $83(48,5 \%)$ & NS \\
\hline Sexo M/F & $29 / 14$ & $75 / 96$ & 0,006 \\
\hline Anemia $(\mathrm{Hb}<10 \mathrm{~g} / \mathrm{dL})$ & $28 / 41(68,2 \%)$ & $74 / 157(47,1 \%)$ & 0,016 \\
\hline Hipercalcemia $(>10,5 \mathrm{mg} / \mathrm{dL})$ & $20 / 36(55,5 \%)$ & $26 / 137(18,9 \%)$ & $<0,001$ \\
\hline Insuficiencia renal (creat >2 mg/dL) & $21 / 40(52,5 \%)$ & $38 / 131(29 \%)$ & 0,006 \\
\hline Diálisis & $8 / 40(20 \%)$ & $10 / 131(7,6 \%)$ & 0,022 \\
\hline Trombocitopenia (<100.000/ $\mu \mathrm{L})$ & $7 / 37(18,9 \%)$ & $10 / 148(6,7 \%)$ & 0,027 \\
\hline \% Plamocitosis medular & $50 \%$ & $37 \%$ & NS \\
\hline Albuminemia $<3,5 \mathrm{~g} / \mathrm{dL}$ & $29 / 34(85,2 \%)$ & $57 / 125(45,6 \%)$ & $<0,001$ \\
\hline Beta-2-microglobulina 3,5 >mg/L & $24 / 33(72,7 \%)$ & $30 / 61(49,1 \%)$ & 0,028 \\
\hline Lesiones líticas múltiples & $19 / 30(63,3 \%)$ & $72 / 118(61 \%)$ & NS \\
\hline Etapa D\&S & & & * \\
\hline I & $1(2,3 \%)$ & $15(8,7 \%)$ & \\
\hline II & $5(11,6 \%)$ & $23(13,4 \%)$ & \\
\hline III & $34(79 \%)$ & $100(58,4 \%)$ & \\
\hline Sin información & $3(6,9)$ & $33(19,3 \%)$ & \\
\hline
\end{tabular}

Las variables se expresan como mediana o número (porcentaje) según corresponda.

NS: no significativo

*No se realizó comparación por elevado número de pacientes sin información.

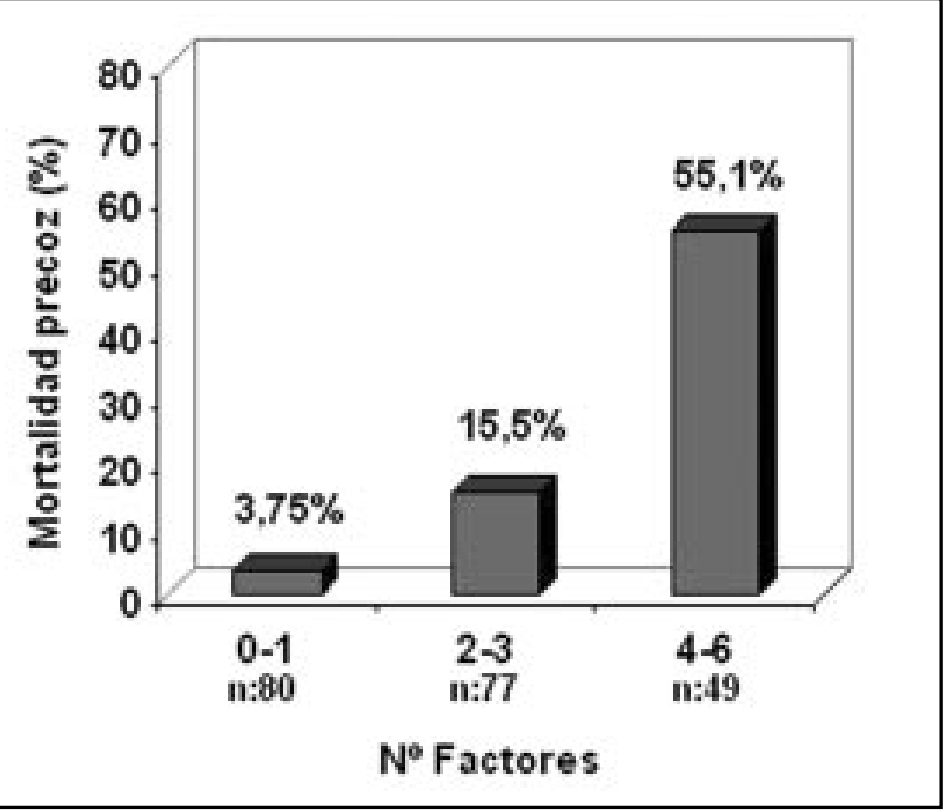

Figura 3. Mortalidad precoz $(<6$ meses desde el diagnóstico) según el número de factores predictores presentes en cada paciente. Se consideraron todos los factores identificados en la Tabla 2 que alcanzaron significación estadística, a excepción de beta-2microglobulina que fue no fue incluida por la alta proporción de pacientes sin información. 
res de 50 años y una mediana de 66 años de edad, similar a la del presente estudio. Esto puede ser explicado por sesgo, incluyendo el presente estudio, al ser estudios basados en centros de referencia y no poblacionales ${ }^{11}$. Hasta el momento no existen estudios poblacionales que sugieran un aumento en la incidencia de $\mathrm{MM}$ en pacientes jóvenes.

La literatura internacional informa una relación de hombres y mujeres de $1,4: 1^{9}$, distinta a la encontrada en este estudio (1:1,1), diferencia que puede sugerir un sesgo en la muestra de pacientes estudiados.

En relación al estudio de laboratorio (Tabla 1), la anemia fue la alteración de laboratorio más frecuente, que era severa $(\mathrm{Hb}<10 \mathrm{~g} / \mathrm{dL})$ en la mitad de los pacientes, lo cual puede estar asociado a diversos factores propios del MM y a una alta frecuencia de insuficiencia renal (30\%). La frecuencia de otras alteraciones de laboratorio es similar a la reportada en la literatura, así como la distribución del tipo de inmunoglobulina ${ }^{10,12}$.

Este estudio revela que existe un grupo de pacientes que fallecen precozmente en el curso de su enfermedad, que definimos como la que ocurre antes de 6 meses desde el diagnóstico. Esta situación ha sido también descrita por Augustson et $\mathrm{al}^{5}$ en un análisis combinado de varias series de ensayos clínicos y por Phekoo et $\mathrm{al}^{11}$, en un estudio en población inglesa. Factores como la edad avanzada de los pacientes (mediana 65-70 años), comorbilidades, insuficiencia renal y efectos adversos del tratamiento (inmunosupresión, eventos tromboembólicos) son asociados a esta mortalidad precoz. En el primero de estos estudios ${ }^{5}$, las infecciones, principalmente neumonías, son la causa más frecuente de muerte durante este periodo, con $45 \%$ de los casos. La mayor susceptibilidad de los pacientes con MM a las infecciones probablemente es multifactorial. Se ha planteado dentro de esto el hecho que los pacientes presentan una síntesis disminuida de inmunoglobulinas policlonales ${ }^{11}$. El tratamiento con glucocorticoides y la aparición de neutropenia secundaria a quimioterapia son otros importantes factores de riesgo ${ }^{13}$.

El estudio de Augustson et $\mathrm{al}^{5}$ sugiere que, a pesar de los avances en el tratamiento del MM, no hay evidencias de una disminución de la mortali- dad precoz. Medidas como uso profiláctico de antibióticos ${ }^{14} 0$ administración de inmunoglobulina endovenosa ${ }^{15}$ han demostrado utilidad en estudios con un número reducido de pacientes, por lo que su efectividad es incierta.

Diversos factores se han asociado a mortalidad en pacientes con MM, desde variables clínicas (anemia, insuficiencia renal, hipencalcemia, plasmocitosis medular elevada, trombocitopenia, beta2-microglobulina e hipoalbuminemia) ${ }^{16}$ a estudios citogenéticos y de genética molecular ${ }^{17}$. Sin embargo, pocos estudios han explorado los factores asociados a mortalidad precoz. En el estudio de Augustson et $\mathrm{al}^{5}$ los factores que predicen esto fueron edad avanzada, bajo sperformance status» y valores elevados de beta2-microglobulina. De nuestro análisis univariado predicen el sexo masculino, anemia, hipencalcemia, insuficiencia renal, trombocitopenia, valores elevados de beta2microglobulina e hipoalbuminemia. Aunque ninguna variable tiene un alto valor predictivo por sí sola, la combinación de factores se asoció a mayor mortalidad precoz (Figura 3). El valor pronóstico de los niveles de beta2-microglobulina y albuminemia es coincidente con el recientemente publicado Sistema de Etapificación Internacional (International Staging System for Multiple Myeloma) ${ }^{18}$. En este, se registraron 10.750 pacientes y pudo establecer que dos variables de laboratorio (beta2-microglobulina y albuminemia) son importantes factores pronóstico de sobrevida de los pacientes con $\mathrm{MM}$.

Las limitaciones en el presente estudio son: análisis retrospectivo de datos, información incompleta en una proporción de los casos y manejo de la totalidad de los casos en centros de referencia. Todo esto nos plantea la necesidad de realizar un estudio prospectivo epidemiológico y considerar este trabajo sólo como el primer intento de conocer la realidad epidemiológica del MM en nuestro país.

\section{Agradecimientos}

Agradecemos al Profesor Doctor Jesús San Miguel, Director del Departamento de Hematología de la Universidad de Salamanca, por el diseño del sistema de registro y al Profesor Doctor Rafael Fonseca, Site Director for Hematological Malignancies, Mayo Clinic Scottsdale, por la revisión y sugerencias sobre el manuscrito. 


\section{REFERENCIAS}

1. Kyle RA, Rajkumar SV. Multiple Myeloma. N Engl J Med 2004; 351: 1860-73.

2. GLOBOCAN 2002 project. International Agency for Research on Cancer. http://www-dep.iarc.fr/ [Consultado el 30 de marzo de 2006].

3. Dispenzieri A, Lacy M, GreipP P. Multiple Myeloma. En: Greer JP, Foerster J, Lukens JN, Rodgers GM, Paraskevas F, Glader BE (eds). Wintrobe's Clinical Hematology, $11^{\text {th }}$ Edition. Lippincot Williams and Wilkins, 2004; 2583-2636.

4. Anderson KC, Pazdurn R, Farrell AT. Development of Effective New Treatments for Multiple Myeloma. J Clin Oncol 2005; 23: 7207-11.

5. Augustson BM, Begum G, Dunn J, Barth NJ, Davies F, Morgan G ET AL. Early Mortality after diagnosis of multiple myeloma: Analysis of patients entered onto the United Kingdom Medical Research Council Trials between 1980 and 2002 -Medical Research Council Adult Leukaemia Working Party. J Clin Oncol 2005; 23: 9219-26.

6. Hungria VTM, Maiolno A, Martínez G, Coelho EOM, Bittencourt R, Souza CA ET AL. South american multiple myeloma study: Epidemiological and clinical characteristics of 751 patients. Haematologica 2005; S1: 120 (abstract po.521).

7. International Myeloma Working Group. Criteria for the classification of monoclonal gammopathies, multiple myeloma and related disorders: a report of the International Myeloma Working Group. Br J Haematol 2003; 121: 749-57.

8. DuRie BGM, Salmon SE. A clinical staging system for multiple myeloma: Correlation of measured myeloma cell mass with presenting clinical features, response to treatment, and survival. Cancer 1975; 36: 842-54.

9. Sirohi B, Powles R. Epidemiology and outcomes research for MGUS, myeloma and amyloidosis. Eur J Cancer 2006; 42: 1671-83.

10. Kyle RA, GerTz MA, Witzig TE, Lust JA, Lacy MQ, DisPENZIERI A ET AL. Review of 1027 patients with newly diagnosed multiple myeloma. Mayo Clin Proc 2003; 78: 15-17.

11. Phekoo KJ, Schey SA, Richards MA, Bevan DH, Bell S, Giliett D ET AL. A population study to define the incidence and survival of multiple myeloma in a National Health Service Region in UK. Br J Haematol 2004; 127: 299-304.

12. Blade J, Rosinol L Renal, hematologic and infectious complications in multiple myeloma. Best Pract Res Clin Haematol 2005; 18: 635-52.

13. Cesana C, Nosari AM, Kiersy C, Miqueileiz S, Rossi V, FERRANDO P ET AL. Risk factors for the development of bacterial infections in multiple myeloma treated with two different vincristine-adriamycin-dexamethasone schedules. Haematologica 2003; 88: 1022-8.

14. Oken MM, Pomeroy C, Weisdorf D, Bennett JM. Prophylactic antibiotics for the prevention of early infection in multiple myeloma. Am J Med 1996; 100: 624-8.

15. Chapel HM, Lee M, Hargreaves R, Pamphilon DH, Prentice AG. Randomized trial of intravenous immunoglobulin as prophylaxis against infection in plateau-phase multiple myeloma. The UK Group for Immunoglobulin Replacement Therapy in Multiple Myeloma. Lancet 1994; 343: 1059-63.

16. San Miguel JF, García-Sanz R. Prognostic features of multiple myeloma. Best Pract Res Clin Haematol 2005; 18: 569-83.

17. Stewart AK, Fonseca R. Prognostic and therapeutic significance of myeloma genetics and gene expression profiling. J Clin Oncol 2005; 23: 6339-44.

18. Greipp PR, San MiguelJ, Durie BG, Crowley JJ, Barlogie B, BLADÉ J ET AL. Intemational staging system for multiple myeloma. J Clin Oncol 2005; 23: 3412-20. 